\title{
Parámetros bio-económicos de la producción intensiva de la carne de bovino en México
}

\section{Bio-economic parameters of intensive production of beef in Mexico}

\author{
Nicolás Callejas-J uáreza , Samuel Rebollar-Rebollarb*, J uan Ángel Ortega-Gutiérreza , \\ Joel Domínguez-Viveros ${ }^{a}$
}

\begin{abstract}
RESUMEN
El objetivo de esta investigación fue evaluar la productividad de carne de bovino en confinamiento estabulado en México, a través de criterios de eficiencia, eficacia, calidad y economía, en cinco entidades (Nuevo León, Tamaulipas, Coahuila, Sonora y Baja California); considerando cinco escalas de producción (desde 3,000 a 7,000 cabezas finalizadas por ciclo). El peso vivo inicial promedio fue $267.00 \pm 35.99 \mathrm{~kg}$ y final $470 \pm 11.73 \mathrm{~kg}$. El ciclo productivo promedio fue de $136 \pm 22$ días y $2.67 \pm 0.34$ ciclos por año. La eficiencia fue de $7.84 \pm 1.39 \mathrm{~kg}$ alimento $/ \mathrm{kg}$ y $61.20 \pm 0.84 \%$ de carne de rendimiento en canal, eficacia $99.55 \pm 0.26 \%$, calidad $100 \%$ y economía $3.36 \pm 1.89 \$ / \mathrm{kg}$ de ingreso neto. La relación beneficio-costo fue de $1.08 \pm 0.03$, con una inversión marginal promedio de 17,815.98 \pm 4,795.81 pesos por cabeza. La conclusión es que la actividad económica presenta una productividad promedio de $63.50 \pm 15.20 \%$.
\end{abstract}

PALABRAS ClAVE: Productividad de carne, Eficiencia, Calidad, Beneficio-costo.

\begin{abstract}
The objective of this research was to evaluate the productivity of beef in confinement in Mexico, through criteria of efficiency, effectiveness, quality and economy, in five entities (Nuevo Leon, Tamaulipas, Coahuila, Sonora and Baja California); considering five scales of production $(3,000,5,000,6,000,7,000$ and 7,500 bovines completed per cycle). The average initial live weight was $267.00 \pm 35.99 \mathrm{~kg}$ and $470 \pm 11.73 \mathrm{~kg}$. The average production cycle was $136 \pm 22 \mathrm{~d}$ and $2.67 \pm 0.34 \mathrm{cycles}$ per year. Efficiency was $7.84 \pm 1.39 \mathrm{~kg}$ feed $/ \mathrm{kg}$ and $61.20 \pm 0.84 \%$ of carcass yield, efficiency $99.55 \pm 0.26 \%, 100 \%$ quality and economy $3.36 \pm 1.89 \$ / \mathrm{kg}$ of net income. The benefit-cost ratio was $1.08 \pm 0.03$, with a marginal investment average of $17,815.98 \pm 4,795.81$ Mexican pesos per bovine. The conclusion is that the economic activity presents an average productivity of $63.50 \pm 15.20 \%$.
\end{abstract}

KEY WORDS: Beef productivity, Efficiency, Meat quality, Benefit-cost.

Recibido el 28 de septiembre de 2015. Aceptado el 27 de noviembre de 2015.

a Facultad de Zootecnia y Ecología. Universidad Autónoma de Chihuahua, Periférico Francisco R. Almada Km 1, 31453. Chihuahua, México.

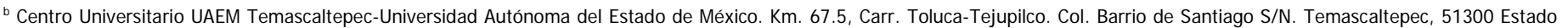
de México. México.

*Autor de correspondencia: srebollarr@uaemex.mx. 


\section{INTRODUCCIÓN}

La relación entre insumos utilizados (naturales, humanos y monetarios) y el producto obtenido, son elementos clave en la toma de decisiones de invertir en el sector agropecuario. La productividad se define como la relación entre la cantidad producida y los recursos utilizados ${ }^{(1,2,3)}$, cuantificados a través de la eficiencia, eficacia, calidad y economía de los sistemas de producción. Otros ${ }^{(4,5)}$, la definen como una actitud orientada hacia el mejoramiento continuo. La eficiencia técnica, es un proceso de producción que no utiliza más insumos de los necesarios para obtener un nivel dado de producción, dada la tecnología existente, y eficiencia económica como un proceso donde la proporción de utilización de los recursos para una determinada tasa de producción, implica el costo mínimo posible ${ }^{(6)}$.

La producción de carne se define como aquellos animales de granja, típicamente un novillo o novilla joven, destinados al sacrificio y confinados hasta su maduración biológica o peso óptimo de mercado; considerando sistemas extensivos, semi-intensivos e intensivos. Se define también como sistemas de producción en pastoreo o confinamiento que hacen hincapié en el crecimiento y desarrollo de los animales sobre la deposición de grasa ${ }^{(7)}$. La producción de carne en corral, implica la provisión de un ambiente artificial en el que los animales se colocan en un área confinada y obligados a consumir una dieta pre-determinada para el propósito de la producción ${ }^{(8)}$. En sistemas de producción estabulados, el de bovinos carne utiliza 3.70 veces menos alimento que en pastoreo para producir la misma cantidad de carne; sin embargo, los porcentajes de concentrados van desde $2 \%$ para sistemas de pastoreo, $4 \%$ para los mixtos y $18 \%$ para los industriales ${ }^{(9)}$.

La eficiencia en producción de carne estabulada, se evalúa con criterios de eficiencia en conversión alimenticia, pero se limita por la correlación entre peso vivo y ganancia promedio diaria ${ }^{(10)}$; la económica, por la capacidad de hacer sus operaciones rentables ${ }^{(6,11)}$ y, la utilidad, por el costo, valor de animales finalizados y costo de alimentación ${ }^{(12)}$. Para México, se consideran además, mano de obra y energéticos (diésel, gasolina y electricidad).

Los métodos utilizados para analizar la rentabilidad y productividad son la regresión matemática, que permite medir la relación (a través del signo) y, la cuantía (a través del coeficiente de regresión) de cada uno de los insumos utilizados; la forma contable, donde al ingreso total se le resta el costo total y, el análisis de regresión estocástica que utiliza el factor riesgo ${ }^{(13,14)}$. Por ello, es importante conocer los resultados de las actividades productivas para poder delimitar su capacidad productiva y de los recursos que intervienen en ella.

En 2013, la eficiencia promedio de la producción de carne de bovino en México, fue $52.7 \%$; se requirió producir $1.9 \mathrm{~kg}$ de carne en pie por cada kilo de carne en canal; esta eficiencia se ha mantenido desde el año 2000. En ese año, la entidad más eficiente fue Baja California (59.9\%) aunque aportó sólo $4.85 \%$ al total nacional, ubicado en 1.8 millones de toneladas de carne en canal(9).

El objetivo de esta investigación fue medir los parámetros de la productividad, en términos de eficiencia, eficacia, calidad y economía, de la producción de carne de bovinos estabulados; a través de cinco escalas de producción (cabezas) ubicadas en los cinco principales estados productores de la República Mexicana.

\section{MATERIAL Y MÉTODOS}

\section{Material}

La investigación se realizó con datos productivos provenientes de cinco unidades representativas de producción (URP) del sistema bovino para carne intensivo, ubicadas en los estados de Nuevo León, Tamaulipas, Coahuila, Sonora y Baja California, en el año de 2010. En total se evaluó la productividad de 54,457 bovinos de diferentes cruzas de razas europeas, $70 \%$ hembras y $30 \%$ machos (Cuadro 1). En total participaron con su información 34 productores; seis productores en Baja California, cinco en Coahuila, ocho en Nuevo León, cinco en Sonora y diez en Tamaulipas. La dieta diaria ( $\mathrm{kg} / \mathrm{día}$ ) estuvo compuesta, en promedio, por cuatro ingredientes principales: maíz rolado (49 \pm 
Cuadro 1. Características de las unidades representativas de producción

\begin{tabular}{lccccc}
\hline Característica/Escala & Sonora & Tamaulipas & Baja California & Nuevo León & Coahuila \\
\hline Escala, cabezas & 7,000 & 7,500 & 3,000 & 6,000 & 5,000 \\
Peso inicial, $\mathrm{kg}$ & 270 & 250 & 300 & 215 & 300 \\
Peso final, kg & 470 & 450 & 475 & 480 & 475 \\
Ciclo productivo, días & 120 & 160 & 160 & 120 & 120 \\
Alimento, kg/cabeza & 2.75 & 2.42 & 2.85 & 2.24 & 2.56 \\
Machos, \% & 40 & 20 & 20 & 30 & 40 \\
Hembras, \% & 60 & 80 & 80 & 70 & 60 \\
Rendimiento en canal, \% & 61 & 62 & 61 & 62 & 60 \\
Precio en pie, $\$ / \mathrm{kg}$ & 21.0 & 22.39 & 20.84 & 21.15 & 20.0 \\
Precio en canal, \$/kg & 34.0 & 36.0 & 34.0 & 34.0 & 32.15 \\
\hline
\end{tabular}

Cabezas de ganado engordado por año: Sonora 3,000; Tamaulipas 5,000; Baja California 6,000; Nuevo León 7,000; Coahuila 7,500.

$12 \%)$, forraje $(20 \pm 7 \%)$, granos secos de destilería (DDG) $(13 \pm 0.5 \%)$, melaza $(8 \pm 6 \%)$ y otros $(10 \%)$.

La obtención de información primaria, se realizó mediante consenso directo de los productores sobre la escala de producción típica de cada una de las entidades, a la cual se le denominó unidad representativa de producción (URP). La URP se definió como el conjunto de productores que tiene características en común, como mercado de insumos y de producto, ubicación de la unidad de producción (en este caso entidad federativa), escala de producción (número de cabezas producidas por año), tecnificación (inversión por cabeza en producción) y ciclo productivo (días del ciclo productivo), principalmente. En todos los casos, la alimentación y peso vivo inicial de los animales fue común y diferente en insumos propios de la región, aprovechando la diferencia de precios. Por ejemplo, en el estado de Tamaulipas los productores sustituyen el sorgo por el maíz; este último se presenta con mayor prevalencia en productores medianos y pequeños.

Los datos de producción se capturaron en una hoja de cálculo en el programa Microsoft Excel 13 para Windows.

\section{Métodos}

Existen varios métodos para medir la productividad como el método de la productividad total, método basado en el tiempo de trabajo, método financiero, productividad del trabajo, método estructural de Kurosawa, método de Lawlor, método de Gold, método de evaluación rápida de la productividad y análisis envolvente de datos (data envelopment analisys $=\mathrm{DEA})^{(4)}$.

Dado que en esta investigación, solo se evaluó la productividad de un solo año, estos métodos resultaron inoperantes, debido a que requieren de al menos dos periodos de evaluación para generar un índice de productividad.

En esta investigación, la productividad se midió a través de indicadores de eficiencia, eficacia, calidad y economía, para, finalmente, obtener un indicador global o total de la actividad. Un indicador, es un valor, medio o instrumento que ayuda a medir, evaluar y tomar decisiones objetivas de un proceso productivo.

La eficiencia (EFI), se midió como la relación entre la cantidad de producto obtenido (Q) y la cantidad de insumos utilizados $\left(\mathrm{x}_{\mathrm{i}}\right)$. Los parámetros técnicos de eficiencia más importantes, fueron el volumen producido por cabeza de ganado, como la diferencia entre el peso vivo final (PVF) y peso vivo al inicio del proceso productivo (PVI); la mortalidad, como la razón entre el número de animales muertos y total de animales en producción; la ganancia de peso, como la razón entre el volumen de carne producido y el número de días del ciclo productivo; y la conversión alimenticia, como la razón entre la 
cantidad de kilogramos consumidos por ciclo y el volumen producido de carne. La eficacia (EF) se definió como el grado de congruencia entre objetivos organizacionales y resultados observables $^{(15)}$. La eficacia significa medir la contribución de cada insumo al logro de los objetivos. Ésta se midió como la diferencia entre la eficiencia, calidad y economía total y la obtenida en el proceso productivo $\left(\mathrm{y}_{\mathrm{i}}\right)$. La calidad (CAL) se midió como la aceptación del producto en el mercado, a través del porcentaje de peso de la canal (PC).

Para este caso, se consideró aceptable por el productor una canal igual o mayor al $60 \%$ del peso vivo (PV). La economía (ECON), se midió como el cumplimiento del objetivo de una inversión, obtener ganancia (G). Esta condición solamente se cumple cuando el ingreso total (IT) es mayor que el costo total (CT); sin embargo, podrían presentarse dos resultados más; que el ingreso total sea igual al costo total $(\mathrm{IT}=\mathrm{CT})$, lo cual supondría el no cumplimiento de la eficacia, e ingreso total menor que costo total IT $<$ CT significa una pérdida de inversión. El ingreso total se obtuvo de multiplicar el volumen producido de carne en canal $\left(Q_{j}\right)$ por el precio de la canal $\left(P_{j}\right)\left(\sum_{j=1}^{m} Q_{j} P_{j}\right)$, y el costo total como la sumatoria de las cantidades de insumo utilizados $\left(Q_{i}\right)$ por su precio $\left(P_{i}\right)\left(\sum_{I 01}^{n} Q_{i} * P_{i}\right)$.

La productividad (PROD) se definió como la efectividad total del sistema de producción. Ésta se midió a través de la suma de la eficiencia (Cuadro 2), eficacia, calidad y economía (Cuadro 3); así, $\mathrm{PROD}=\mathrm{EFI}+\mathrm{EF}+\mathrm{CAL}+\mathrm{ECON}$. El criterio de análisis estadístico para evaluar la diferencia entre productores, dentro de cada parámetro productivo, fue a través de su media y desviación estándar $(\bar{x} \pm \sigma)$.

Para evaluar la productividad, se utilizaron 15 indicadores de eficiencia, eficacia, calidad y economía, que caracterizan a un sistema de

Cuadro 2. Parámetros de eficiencia de las unidades representativas de producción

\begin{tabular}{lccccc}
\hline Parámetro/Escala & Sonora & Tamaulipas & Baja California & Nuevo León & Coahuila \\
\hline Capacidad utilizada, \% & 100 & 60 & 50 & 100 & 67 \\
Producción, kg & 175 & 175 & 265 & 200 & 200 \\
Ganancia diaria de peso, kg & 1.09 & 1.46 & 2.21 & 1.67 & 1.25 \\
Relación machos-hembras & $20-80$ & $40-60$ & $30-70$ & $40-60$ & $20-80$ \\
Conversión alimenticia, kg & 9.54 & 7.36 & 7.22 & 6.12 & 8.97 \\
Ciclos/año & 2.6 & 3.0 & 3.0 & 2.5 & 2.2 \\
Mortalidad, \% & 0.26 & 0.40 & 0.33 & 0.24 & 0.35 \\
Inversión, \$/kg & 19.54 & 19.69 & 19.03 & 18.23 & 19.46 \\
\hline
\end{tabular}

Cuadro 3. Parámetros económicos de las unidades representativas de producción

\begin{tabular}{lccccc}
\hline Parámetro/Escala & Sonora & Tamaulipas & Baja California & Nuevo León & Coahuila \\
\hline Subsidio, \% inversión & 0.43 & 0.85 & 0.72 & 0.93 & 0.46 \\
Ingreso, \$/kg & 21.59 & 20.80 & 22.03 & 21.91 & 23.17 \\
Costo, \$/kg & 20.35 & 19.00 & 21.02 & 19.31 & 21.61 \\
Ingreso neto, \$/kg & 1.25 & 1.80 & 1.01 & 2.60 & 1.57 \\
Relación beneficio/costo & 1.06 & 1.09 & 1.05 & 1.13 & 1.07 \\
Precio, \$/kg en pie & 20.84 & 20.00 & 21.15 & 21.00 & 22.39 \\
Precio, \$/kg en canal & 34.00 & 32.15 & 34.00 & 34.00 & 36.00 \\
Inversión, \$/kg & 19.54 & 19.69 & 19.03 & 18.23 & 19.46 \\
Rendimiento/inversión, \% & 6.38 & 9.16 & 5.30 & 14.27 & 8.05 \\
\hline
\end{tabular}


producción de carne de bovino en confinamiento (Cuadro 4). La escala de producción (animales en engorda por año), con la mejor medición, se le asignó el número 5; la escala de producción con la peor medición, se le asignó el 1 . En términos porcentuales, el número 5 correspondió a $100 \%$ y el número 1 al $20 \%$.

Finalmente, el factor riesgo está asociado fuertemente con este sistema de producción. En esta investigación se evaluaron los parámetros mortalidad, eficiencia de la alimentación, costo de producción, ingreso y rentabilidad, utilizando el programa estadístico Simetar ${ }^{\circledR}$, complemento de Excel $2013^{(14)}$. Se utilizó un modelo de simulación del riesgo con una distribución GRKS. La distribución GRKS, es una de dos piezas de distribución normal con $50 \%$ del peso por debajo del valor medio, $2.5 \%$ menos que el mínimo, $50 \%$ por encima del valor medio y $2.5 \%$ por encima del máximo. La distribución se utiliza en lugar de una distribución triángulo cuando se sabe solamente información mínima sobre la variable aleatoria y el mínimo y máximo son inciertos ${ }^{(14)}$. La distribución GRKS se basa en la simulación de una distribución empírica.

$$
\text { Probabilidad }=\operatorname{EMP}\left(\mathrm{X}_{\mathrm{i}}, \mathrm{F}\left(\mathrm{X}_{\mathrm{i}}\right) \text {, CUSD }\right)
$$

Donde EMP, es la distribución empírica, $X_{i}$ son los datos de la variable a simular, $F\left(X_{i}\right)$ es el porcentaje de desviaciones de la tendencia ("percentage deviations from trend") que toma valores entre cero y uno, CUSD son las desviaciones estándar uniformes correlacionadas ("correlated uniform standard deviates").

\section{RESULTADOS Y DISCUSIÓN}

Las características más importantes que definieron al sistema de producción de carne de bovino confinado en México, fueron la composición de la dieta alimenticia, a base de maíz rolado y minerales $(100 \%)$, pasto de la región $(80 \%)$, melaza ( $80 \%$ ), harinolina (60\%) y sebo ( $40 \%)$; el sexo, principalmente hembras ( $70 \%)$, el destino de los animales finalizados, $100 \%$ de los productores vendieron en canal para aprovechar el precio y transferencia gubernamental por sacrificar en rastro TIF (únicamente machos), porque representó un incremento del ingreso, a la vez que se eliminó un intermediario y se evaluó el peso de las canales en frío.

Cuadro 4. Parámetros de productividad de las unidades representativas de producción

\begin{tabular}{lccccc}
\hline Parámetro/Escala & Sonora & Tamaulipas & Baja California & Nuevo León & Coahuila \\
\hline Capacidad utilizada, \% & 5 & 3 & 2 & 5 & 4 \\
Volumen producido, kg & 1 & 3 & 4 & 5 & 2 \\
Alimento, kg/cabeza & 3 & 4 & 1 & 5 & 2 \\
Eficiencia, kg/kg* & 1 & 3 & 4 & 5 & 2 \\
Costo alimento** $_{\text {Ingreso/animal, \$ }}^{*}$ & 1 & 5 & 3 & 4 & 2 \\
Costo/animal, \$ & 1 & 2 & 5 & 3 & 4 \\
Ingreso neto, \$/kg & 3 & 5 & 1 & 4 & 2 \\
RBC & 2 & 4 & 1 & 5 & 3 \\
Rendimiento canal, \% & 2 & 4 & 1 & 5 & 3 \\
Precio, \$/kg en pie & 4 & 3 & 5 & 4 & 5 \\
Precio, \$/kg en canal & 2 & 1 & 4 & 3 & 5 \\
Inversión, \$/kg & 4 & 3 & 4 & 4 & 3 \\
Ganancia/inversión, \% & 2 & 1 & 4 & 5 & 3 \\
Mortalidad, \% & 2 & 4 & 1 & 5 & 4 \\
\hline
\end{tabular}

*Kilogramos de carne/kilogramos de alimento.

** \$/cabeza.

$\mathrm{RRS}=$ relarińn henefirin/rnstn 
Con relación al destino de la carne, $100 \%$ de los productores tiene como principal mercado el nacional; sin embargo algunos productores (15\%) reportaron exportaciones de cortes de mayor valor (Sukarne, Procarne, entre otros). El $100 \%$ de los productores aplicó implantes, debido a la baja calidad del ganado engordado. El $100 \%$ de los productores utilizó cinco etapas de producción; todos los productores presentaron problemas de mortalidad en su hato en la primera etapa de la producción, debido a que los animales no se adaptaron rápidamente al tipo de alimento (ingredientes) y hábitos de consumo (tres veces al día).

La eficiencia productiva entre machos y hembras se consideró como de mínima importancia entre los productores, y solamente se vio reflejada en la transferencia gubernamental al sacrificio de machos (200 \$/cabeza). Sin embargo, se encontró que no existe una diferencia significativa ( $P>0.05)$ en el peso vivo promedio inicial y final; esto significa que animales con menor peso tuvieron un mayor rendimiento, y fue la razón por la que el productor prefirió becerros livianos. Este resultado coincide con el de otras investigaciones, donde se indica que el costo de agregar peso vivo es, generalmente, menor que el costo de comprar animales con mayor peso vivo ${ }^{(11)}$. Esto explica por qué el costo promedio de producción está representado por tres rubros, compra de ganado $52.5 \%$, alimentación $40.3 \%$ y otros $7.2 \%$; es decir, el productor asume principalmente dos tipos de riesgo, el primero en comprar la materia prima adecuada (animales), y segundo, la alimentación, si no se considera mano de obra, combustibles e instalaciones.

\section{Eficiencia}

Una de los aspectos más importantes en la producción de carne bajo el sistema estabulado intensivo es la relación machos-hembras 30-70. Sin embargo, esto es consecuencia de la exportación de los becerros machos; mientras que en la producción, la diferencia de rendimiento, para los productores, es mínima. En otros hallazgos ${ }^{(12)}$, se encontró que la ganancia diaria de peso y conversión alimenticia entre machos castrados y hembras fueron significativas $(P<0.05)$; también, la ganancia de peso diario fue mejor en hembras que en machos castrados, no así la conversión alimenticia.

En promedio, la capacidad utilizada fue $75.40 \pm$ $23.3 \%$ y $2.67 \pm 0.34$ ciclos productivos por año. Una de las causas que se le atribuye a esta ineficiencia, es la disponibilidad de ganado; como consecuencia de la exportación a los Estados Unidos, los productores tratan de resolver este problema engordando las hembras.

El volumen producido por cabeza de ganado fue de $203 \pm 36.84 \mathrm{~kg}$ de peso vivo (sin considerar el PV inicial), en un periodo de $136 \pm 22$ días, representó $1.54 \pm 0.43 \mathrm{~kg} /$ día de rendimiento. No obstante, solamente $40 \%$ de los productores obtuvieron un rendimiento superior al promedio (Baja California y Nuevo León).

La mortalidad promedio, fue $0.32 \pm 0.07 \%$. Una de las principales causas de mortalidad es la falta de adaptación que tienen los animales una vez que llegan a los corrales de engorda, la cual se dificulta por provenir de un régimen de alimentación de repasto. La estrategia utilizada por algunos productores ha sido seleccionar los lotes de ganado de acuerdo a su adaptación, esto es, aquellos más adaptados van directamente a los corrales, y el resto pasa por un proceso previo de adaptación. En otros resultados $^{(11)}$, se encontró que la mortalidad anual en la engorda estabulada de bovinos en adaptación fue $0.69 \%$, principalmente de origen tóxico (37\%) y respiratorio (29.9\%); por arriba del promedio en México.

La conversión alimenticia fue $7.84 \pm 1.39 \mathrm{~kg}$ de alimento por kilogramo de carne producido; esta eficiencia coincide con resultados hallados en investigaciones similares $^{(12,16)}$. La ganancia diaria de incremento de peso vivo fue $1.54 \pm 0.43 \mathrm{~kg}$, lo que significa una eficiencia de $0.30,0.36$ y $0.26 \mathrm{~kg}$ por arriba del que se reportó en otras investigaciones $^{(17,18,19)}$ y similar a lo encontrado en un trabajo sobre bovinos engordados en corral en una región de México ${ }^{(20)}$, en que la GDP promedio fue $0.29 \mathrm{~kg}$. Finalmente, para producir un kilogramo de carne en pie se requirió invertir $19.19 \pm 0.26 \$ / \mathrm{kg}$ (Cuadro 2).

Algunas investigaciones realizadas en la engorda de bovinos muestran una eficiencia menor 
y mayor que en México; así, se tiene que en Turquía $^{(12)}$ bovinos finalizados con peso de 450.97 $\mathrm{kg}$ tuvieron una eficiencia en la canal de $55.59 \%$, con $500.98 \mathrm{~kg}$, rendimiento $56.94 \%$ y con 556.15 $\mathrm{kg}$ rendimiento de $59.14 \%$. Análogamente, en Jalisco, México, se encontraron incrementos de peso de $0.945 \mathrm{~kg} /$ día y conversión alimenticia de $11.60 \mathrm{~kg}$ de alimento por kilogramo de carne en pie $^{(20)}$. En Tanzania(21) se encontró que en una engorda de bovinos con peso inicial de 184 y final de $278 \mathrm{~kg}$ por un periodo de 100 días, tuvieron una conversión alimenticia de 8 a 1 . En contraste, en otro hallazgo ${ }^{(19)}$, se demostró que la máxima ganancia en dinero para bovinos en corral fue a los $473.94 \mathrm{~kg}$ PVF y el máximo peso de los animales finalizados fue $475.04 \mathrm{~kg}$ PVF.

\section{Eficacia}

La eficacia se midió utilizando los mismos parámetros de eficiencia, pero en cumplimiento de los objetivos de cada insumo utilizado en la producción. En la capacidad utilizada de corrales, solamente $40 \%$ de los productores la utilizó de manera eficaz; es decir, utilizaron los corrales al $100 \%$; el resto de productores está dejando de producir 20,685 cabezas de ganado equivalentes a 9,736 toneladas de carne en pie o 13.5 millones de pesos de utilidad. En la conversión alimenticia, considerando al mejor (Nuevo León), el resto de los productores está utilizando $8.63 \mathrm{~kg}$ de más, lo que puede producir 1.41 kilogramos adicionales; el productor más eficiente necesitaría $24.48 \mathrm{~kg}$ de alimento para producir un kilogramo de carne, en lugar de $39.21 \mathrm{~kg}$.

\section{Calidad}

El único parámetro de calidad en este sistema de producción fue el rendimiento de la canal, cuyo promedio fue $61.20 \pm 0.84 \%$. Al respecto, en Tanzania(21) se encontró que bovinos engordados por un periodo de 100 días con peso vivo final de $278 \mathrm{~kg}$ obtuvieron un rendimiento de la canal de $55.80 \%$. A su vez, en Canadá, novillos finalizados en corral, a base de maíz, con peso inicial de $\mathbf{2 8 1 . 1 0}$ $\pm 19.60 \mathrm{~kg}$ y final de $634 \mathrm{~kg}$ obtuvieron una eficiencia de la canal de $58.40 \%{ }^{(16)}$.
En Oklahoma, obtuvieron rendimientos de la canal por arriba del encontrado en esta investigación utilizando diferentes razas de ganado Angus $\left(63.70 \%\right.$ ) y Brangus (64.4\%) ${ }^{(17) ;}$ sin embargo, en otra investigación ${ }^{(19)}$, se encontró que para toretes finalizados en corral, el PVF equivalente al nivel óptimo económico fue $473.94 \mathrm{~kg}$ y un rendimiento en canal de $60 \%$. En el caso de las engordas analizadas predominan las cruzas, porque las razas puras se exportan.

\section{Economía}

La inversión requerida en el sistema de producción de carne de bovino estabulada es alta; en promedio se tiene invertido poco más de $97 \pm 30$ millones de pesos, por lo que las transferencias de gobierno (subsidios) solamente representaron 0.68 $\pm 0.23 \%$ de la inversión total, principalmente en la etapa de sacrificio. El ingreso neto promedio fue $1.65 \pm 0.65 \$ / \mathrm{kg}$ en pie. La relación beneficio-costo fue $1.08 \pm 0.03$; significa que por cada peso invertido se obtuvo un rendimiento de $8 \%$, o bien, el ingreso representó 1.08 veces el costo de producción. La inversión promedio por kilogramo producido de carne en pie fue $19.19 \pm 0.59$ pesos. Por cada peso obtenido de ingreso neto se invirtieron $8.63 \pm 3.49$ pesos (Cuadro 3). En hallazgos similares ${ }^{(22)}$, se encontraron $18.41 \$ / \mathrm{kg}$ en ganado finalizado en engorda en corral, resultados cercanos a los de esta investigación.

Por kilogramo producido de carne en pie, el costo económico fue 18.40 pesos, principalmente porque el costo del alimento promedio fue $8.53 \pm$ 1.40 pesos ( $46.40 \%$ ); esto significaría producir 0.91 kilogramos de más. De forma similar, otros investigadores encontraron que el costo de producción fue 16.74 (2011=100) $\$ / \mathrm{kg}$ de carne en pie y un margen de ganancia de $2.50 \quad(2011=100)$ pesos ${ }^{(16,20)}$; lo que concuerda con los resultados obtenidos para una región del Estado de México(22), en la que el costo de producción para productores pequeños fue 20.09, medianos 17.87 y grandes $18.41 \$ / \mathrm{kg}$; mientras que la ganancia de medianos y grandes fue superior con relación a la ganancia que se obtuvo en esta investigación. 
Figura 1. Elementos de la productividad

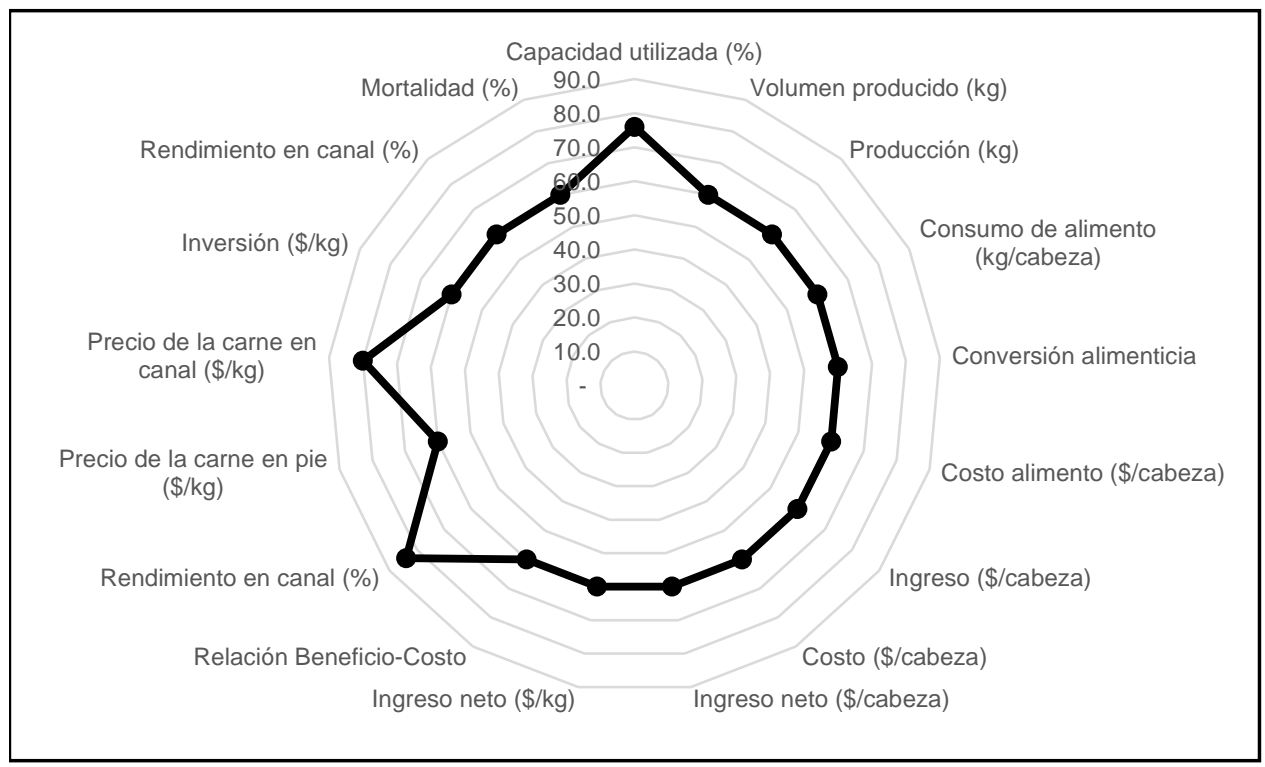

\section{Productividad total}

Considerando 15 indicadores de eficiencia, eficacia, calidad y economía (Cuadro 4); la productividad fue $63.5 \pm 8.0 \%$. La mayor productividad correspondió a Nuevo León con 88.24 $\pm 15.9 \%$, seguido de Tamaulipas ( $63.53 \pm 25.7 \%)$, Coahuila ( $62.35 \pm 24.4 \%$ ), Baja California ( $56.5 \pm$ $32.6 \%$ ) y por último Sonora ( $47.06 \pm 23.4 \%$ ).

Los parámetros más importantes fueron el rendimiento de la canal ( $84 \%)$, el precio de la canal $(80 \%)$ y la capacidad utilizada (76\%); el resto tuvieron una productividad promedio de $60 \%$ (Figura 1).

\section{Riesgo}

El riesgo asociado al costo de producción e ingreso, significa que para una probabilidad de $100 \%$ el costo total sea de $21.61 \$ / \mathrm{kg}$ e ingreso de $27.17 \$ / \mathrm{kg}$; mientras que la probabilidad mínima fue 19 y $20.80 \$ / \mathrm{kg}$, respectivamente; la mayor diferencia entre ingreso y costo se obtuvo con una probabilidad de $30 \%$. Así mismo, la relación beneficio-costo de mayor probabilidad fue 2.20 y mínima 1.09, lo que significa que por cada peso invertido, se obtendrán 1.20 pesos de ganancia como máximo y 0.90 pesos como mínimo (Figura 2a).
Existe una probabilidad de $100 \%$ de que el ingreso neto o utilidad sea $2.60 \$ / \mathrm{kg}$ como máximo y $1.01 \$ / \mathrm{kg}$ como mínimo; esto significa que el sistema de producción es rentable en todas sus escalas de producción (Figura 2b). Para lograr un ingreso neto per cápita de $100 \$ /$ día, el productor más eficiente deberá producir $38.46 \mathrm{~kg}$ y el menos eficiente $99 \mathrm{~kg}$. En este caso la productividad significa $60.54 \mathrm{~kg}$ o bien 157.42 pesos de ingreso neto. La máxima fue $0.40 \%$, mínima $0.24 \%$ y la probabilidad promedio $0.33 \%$ (Figura 2c).

Esto significa que por cada 100 bovinos, se tendrá una mortalidad máxima de 4 cabezas de ganado, mínima de 2.4 y promedio de 3.3 cabezas de ganado. Finalmente, la eficiencia del alimento indica que con una probabilidad de $100 \%$ se tendrá una ganancia de peso diaria máxima de 2.21 $\mathrm{kg} /$ animal y 1.09 como mínima; en promedio, se espera $1.46 \mathrm{~kg} /$ día (Figura 2d). Esto significa que el productor menos eficiente (Sonora) necesitaría 79 días para producir los $175 \mathrm{~kg}$ con una eficiencia del productor más eficiente (Baja California) de 2.21 $\mathrm{kg} / \mathrm{d}$ ía/animal. En conjunto y considerando la máxima eficiencia, se estaría ahorrando 208 días por ciclo productivo. A este respecto, un productor de ganado de engorda se enfrenta a dos tipos de riesgo, la variabilidad de precios de mercado y la 
Figura 2. Factores de riesgo en las unidades representativas de producción

a) Costo e ingreso

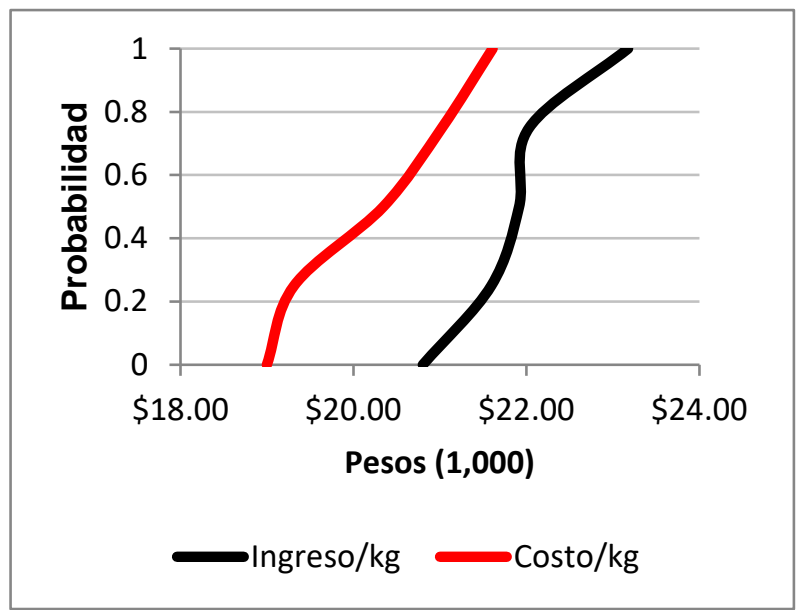

b) Utilidad

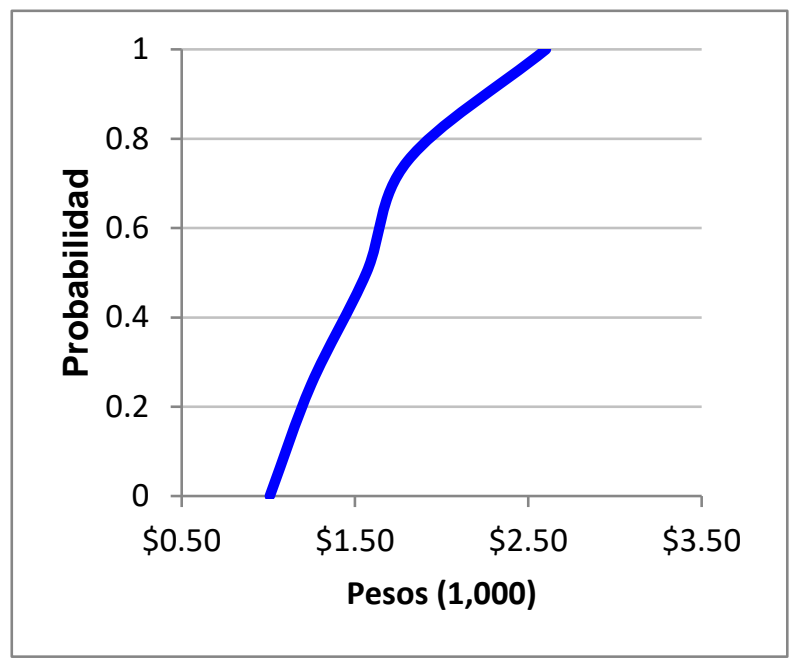

variabilidad de la producción ocasionada por las condiciones ambientales y manejo del ganado ${ }^{(23,24)}$.

\section{CONCLUSIONES E IMPLICACIONES}

La producción de carne de bovino finalizado en confinamiento estabulada en México es rentable; sin c) Mortalidad

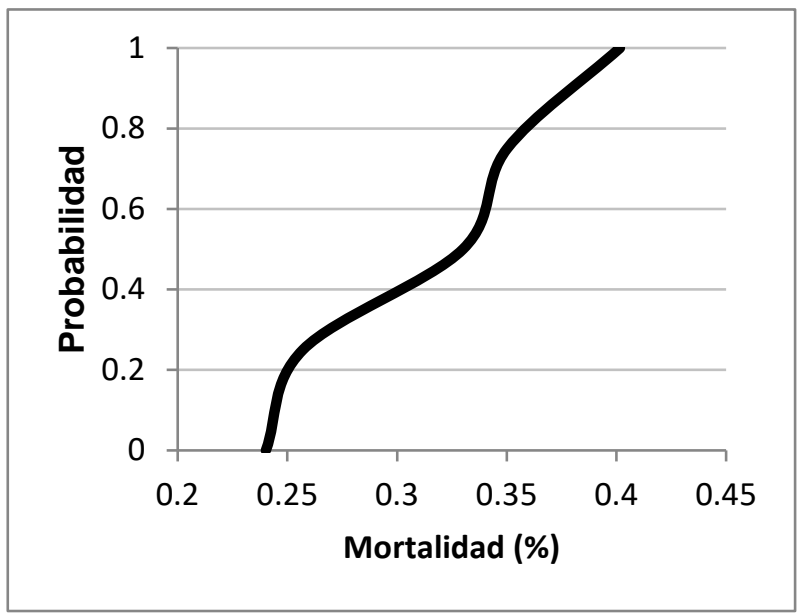

d) Eficiencia del alimento

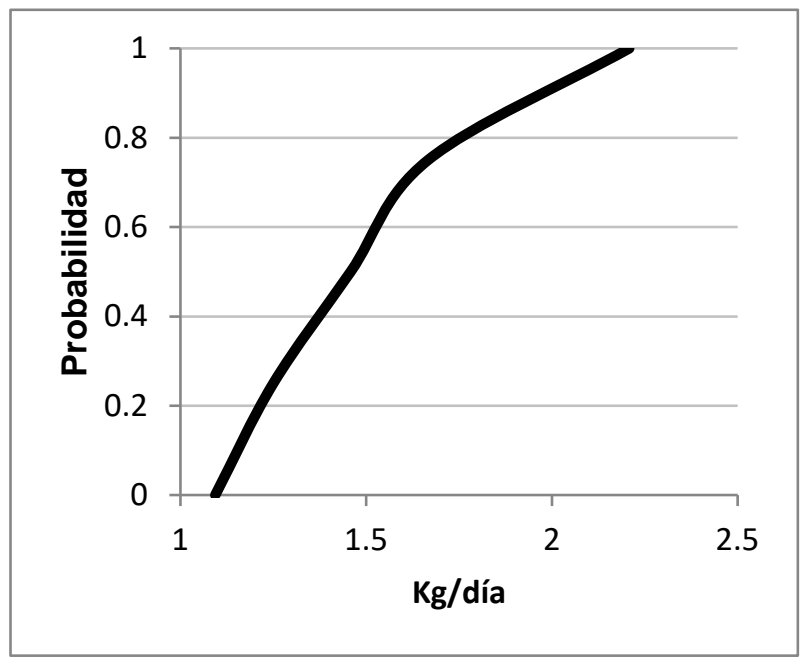

embargo, presenta una productividad promedio de $63.50 \%$. El sistema se caracterizó por finalizar principalmente hembras. Los parámetros que definieron la productividad fueron rendimiento en canal, precio de la canal y capacidad utilizada. Finalmente, el volumen producido de carne de bovino en confinamiento en México es bajo, y está 
Nicolás Callejas-J uárez, et al. / Rev Mex Cienc Pecu 2017;8(2):129-138

determinado por el precio de la carne en canal, capacidad utilizada de las instalaciones y mortalidad.

\section{LITERATURA CITADA}

1. Smith A. An inquiry into the nature and causes of the wealth of nations. 1st ed. London, UK: Methuen \& Co; 1776.

2. Bain D. Productividad: la solución a los problemas de la empresa. J MR y Rodríguez editores. México, DF: McGraw-Hill; 1985.

3. Belcher JG. Productividad total I: cómo aprovechar los recursos para obtener ventaja competitiva. 1ra ed. Buenos Aires, Argentina: Ediciones Granica; 1991.

4. Benicio J, de Mello J CS. Productivity analysis and variable returns of scale: DEA Efficiency Frontier Interpretation. Procedia Comp Sci 2015; (55):341-349.

5. Gerbens-Leenes LPW, Mekonnen MM, Hoekstra AY. The water footprint of poultry, pork and beef: A comparative study in different countries and production systems. Water Resour Industry 2013;(1):25-36.

6. Langemeier $M$, Schroeder $T$, Mintert J. Determinants of cattle finishing profitability. J Agric Applied Economics 1992;24(2):4148.

7. Richardson JW, Outlaw J L, Knapek GMJ, Raulston M, Herbst BK, Anderson DP, Klose SL. Representative farms economic outlook for the December. Agrilife Res Ext. 2012.

8. FAO. Food and Agriculture Organization of the United Nations. Base de datos estadísticos 2014. http://www.fao.org/ag/againfo/ themes/es/meat/home.html. Consultado 24 mar, 2015.

9. SIAP. Sistema de Información Agroalimentaria y Pesquera. Ganadería. http://www.siap.gob.mx/ganaderia/. Consultado 5 Jun, 2015

10. Costa EF, Giuliodori MJ, Dezzilio M, Romero JR. Mortalidad en un feedlot de La Plata (Buenos Aires, Argentina): causas, distribución mensual e impacto económico. Analecta Veter 2003;23(1):13-19.

11. Demircan V. The effect of initial fattening weight on sustainability of beef cattle production in feedlots. Spanish J Agric Res 2008; 6(1):17-24

12. Meissner HH, Smuts M, Coertze RJ . Characteristics and efficiency of fast-growing feedlot steers fed different dietary energy concentrations. J Anim Sci 1995: 73(4): 931-936.
13. Wang X, Dorfman JH, McKissick J, Turner SC. Optimal marketing decisions for feeder cattle under price and production risk. J Agric Applied Economics 2001;33(3):431-443.

14. Richardson JW, Schumann KD, Feldman PA. SI METAR: Simulation and econometrics to analyze risk. College Station, Texas. Simetar, Inc. 2006

15. Santana MHA, Rossi P, Almeida R, Cucco DC. Feed efficiency and its correlations with carcass traits measured by ultrasound in Nellore bulls. Livestock Sci 2012;145(1):252-257.

16. Yang $\mathrm{WZ}, \mathrm{Xu}$ L, Li C, Beauchemin KA. Short communication: Effects of supplemental canola meal and various types of distillers' grains on growth performance of backgrounded steers. Canadian J Anim Sci 2013;93(2):281-286.

17. DeRouen SM, Wyatt WE, Bidner TD, Persica MA. Feedlot and carcass performance of Angus, Brangus, Gelbvieh and Gelbraysired crossbred steers. The Professional Anim Scient 2000; 16(1):6-12.

18. Westhoff P, Brown S. US baseline briefing book: Projections for agricultural and biofuel markets. FAPRI-MU Report - Food and Agricultural Policy Research Institute, College of Agriculture, Food and Natural Resources. University of Missouri 2011;2(11):68.

19. Rebollar RS, Posadas DRR, Hernández MJ, Rojo RR, González RJ, Guzmán SE. Technical and economic optimum in feedlot. Trop Subtrop Agroecosyst 2011;(14):413-420.

20. Álvarez S, Gutiérrez E. Engorda de toretes a base de estiércol fresco de cerdo y dos fuentes de fibra en una empresa comercial. Livest Res Rural Develop 2001;13(4).

21. Asimwe L, Kimambo AE, Laswai GH, Mtenga LA, Weisbjerg MR, Madsen J. Effect of days in feedlot on growth performance, carcass and meat quality attributes of Tanzania shorthorn zebu steers. Trop Anim Health Prod 2015;475):867-876.

22. Rebollar RA, Hernández MJ, Rebollar RS, González RFJ, García MA, Guzmán SE. Competitiveness and profitability of beef cattle in the South of State of Mexico. Trop Subtrop Agroecosyst 2011; 14(2):691-698.

23. Carr, M. Beef cattle feedlots-how to measure, manage and monitor. Bovine medicine. 3rd ed. Washington, USA: John Wiley \& Sons, Ltd; 2015.

24. Peel DS, Mathews Jr KH, Johnson RJ. Trade, the expanding Mexican beef industry, and feedlot and stocker cattle production in Mexico. J Current Issues in Globalization 2011;5(4):475. 